\title{
On Some New Properties of Soft Set Operations
}

\author{
D. Singh \\ (Former Professor, Indian Institute of Technology, \\ Bombay) \\ Mathematics Department \\ Ahmadu Bello University \\ Zaria,Nigeria
}

\author{
Onyeozili, I.A. \\ Department of Mathematics \\ University of Abuja,Nigeria \\ (PhD Research Scholar)
}

\begin{abstract}
In this paper, we establish some new results related to distributive properties of AND $(\wedge)$ and OR $(\vee)$-operations with respect to operations of union, restricted union restricted intersection, extended intersection and restricted difference on soft sets, and provide some illustrative examples.
\end{abstract}

\section{Keywords and Phrases:}

Soft sets operations, AND-operation, OR-operation, Distributivity.

\section{INTRODUCTION}

More often than not, real life problems inherently involve uncertainties, imprecision and vagueness. In particular, such classes of problems arise in economics, engineering environmental sciences, medical sciences, social sciences, etc. In course of time, a number of mathematical theories such as probability theory, fuzzy set theory, rough set theory, interval mathematics theory, vague set theory, etc., formulated to solve such problems, have been found only partially successful.

The major reason for the difficulties arising with the aforesaid theories is due to the inadequacies of their parameterization tools (See Molodtsov [5]).

In order to overcome these difficulties, Molodtsov [5] presented an elaborate theory of soft sets as a completely new mathematical tool with adequate parameterization for dealing with uncertainties. This prototype work of Molodtsov, besides unfolding a rich potential of soft set theory for applications, included directions for further research, especially discovering new operations on soft sets and their properties ([5], p. 21). It is encouraging to note that a number of researches in this direction have appeared during the labasic algebraicbb st few years.

Following the work of Molodtsov [5], Maji et al. [4] were the first to present a detailed study of soft set and its operations.

Ali et al. [1], Sezgin and Atagun [8], Ge and Yang [3], etc., pointed out some modifications in the work of Maji et al. [4]. They also introduced some new operations and established some of their properties.Qin and Hong [7] and $\mathrm{Fu} \mathrm{Li} \mathrm{[2]} \mathrm{also}$ established some results concerning these new operations.Neog and Sut[6] and Zhu and Wen[9] redefinded the intersection, complement and difference of soft sets and investigated their basic algebraic properties.

In this paper, we establish some new results on distributive properties of AND $(\wedge)$ and OR $(\vee)$ operations on soft sets with respect to union $(\tilde{U})$, restricted union $\left(\cup_{R}\right)$,

\author{
restricted intersection $(\mathrm{O})$, extended intersection $\left(\cap_{\varepsilon}\right)$ and \\ restricted difference $\left(\begin{array}{l}R \\ R\end{array}\right)$ on soft sets, and provide some \\ illustrative examples.
}

\section{PRELIMINARIES}

Definition 2.1 [3]

Let $U$ be an initial universe se and $E$ be a set of possible parameters with respect to $U$. Let $P(U)$ denote the power set of $U$ and $A \subseteq E$. Then a pair $(F, A)$ is called a soft set over $U$, where $F$ is a mapping given by

$F: A \rightarrow P(U)$.

For $e \in A, F(e)$ may be considered as the set of eapproximate elements of the soft set $(F, A)$. Thus $(F, A)$ can be defined as a set of ordered pairs given by

$$
(F, A)=\{(e, F(e) \mid e \in A \subseteq E, F(e) \in P(U)\} .
$$

\section{Definition 2.2 [3]}

For two soft sets $(F, A)$ and $(G, B)$ over a common universe $U$, we say that:

(1) $(F, A)$ is a soft subset of $(G, B)$ if

(i) $A \subseteq B$, and

(ii) $\forall e \in A, F(e)$ and $G(e)$ are identical approximations.

We write $(\boldsymbol{F}, \boldsymbol{A}) \simeq(\boldsymbol{G}, \boldsymbol{B})$.

(2) $(F, A)$ is soft equal to $(G, B)$,denoted $(F, A)=(G, B)$, if $(F, A)$ is a soft subset of $(G, B)$ and $(G, B)$ is a soft subset of $(F, A)$.

\section{Definition 2.3 [7]}

Let $(F, A)$ and $(G, B)$ be two soft sets over the same universe $U$. Then the AND-operation of $(F, A)$ and $(G, B)$,denoted $(F, A) \wedge(G, B)$, is a soft set $(H, A \times B)$ where $\forall(\alpha, \beta) \in A \times B$,

$$
H(\alpha, \beta)=F(\alpha) \cap G(\beta) \text {. }
$$

\section{Definition 2.4 [7]}

Let $(F, A)$ and $(G, B)$ be two soft sets over the same universe $U$. Then the OR-operation of $(F, A)$ and $(G, B)$, denoted $(F, A) \vee(G, B)$, is a soft set $(H, A \times B)$ where $\forall(\alpha, \beta) \in A \times B$, $H(\alpha, \beta)=F(\alpha) \cup G(\beta)$.

\section{Definition 2.5 [7]}

The union of two soft sets $(F, A)$ and $(G, B)$ over a common universe $\mathrm{U}$ denoted $(\boldsymbol{F}, \boldsymbol{A}) \widetilde{\cup}(\boldsymbol{G}, \boldsymbol{B})$, is the soft set $(H, C)$, where $C=A \cup B$ and $\forall e \in C$, 
$H(e)=\left\{\begin{array}{l}F(e), \text { if } e \in A-B ; \\ G(e), \text { if } e \in B-A ; \text { and } \\ F(e) \cup G(e), \text { if } e \in A \cap B .\end{array}\right.$

\section{Definition 2.6 [5]}

The restricted union of two soft sets $(F, A)$ and $(G, B)$ over a universe $U$, denoted $(F, A) \cup_{R}(G, B)$, is the soft set $(H, C)$ where $C=A \cap B \neq \varnothing$ and $\forall e \in C$,

$$
H(e)=F(e) \cup G(e) .
$$

\section{Definition 2.7 [5]}

The extended intersection of two soft sets $(F, A)$ and $(G, B)$ over a common universe $U$, denoted $(F, A) \cap_{\varepsilon}(G, B)$, is the soft set $(H, C)$ where $C=A \cup B$ and $\forall e \in C$,

$H(e)=\left\{\begin{array}{l}F(e), \text { if } e \in A-B ; \\ G(e), \text { if } e \in B-A ; \text { and } \\ F(e) \cap G(e), \text { if } e \in A \cap B .\end{array}\right.$

\section{Definition 2.8 [5]}

The restricted intersection of two soft sets $(F, A)$ and $(G, B)$ over a common universe $U$, denoted $(F, A) \mathrm{O}(G, B)$, is the soft set $(H, C)$ where $\boldsymbol{C}=\boldsymbol{A} \cap \boldsymbol{B} \neq \varnothing$ and $\forall e \in C$,

$H(e)=F(e) \cap G(e)$.

\section{Definition 2.9 [5]}

The restricted difference of two soft sets $(F, A)$ and $(G, B)$ over a common universe $U$, denoted $(F, A){ }_{R}(G, B)$,is the soft set $(H, C)$ where $C=A \cap B \neq \varnothing$ and $\forall e \in C$,

$H(e)=F(e)-G(e)$

\section{DISTRIBUTIVE PROPERTIES OF AND-OPERATION AND OR- OPERATION ON SOFT SETS}

\section{Proposition 3.1 (Distributive Properties of OR-Operation)}

Let $(F, A),(G, B)$ and $(H, C)$ be soft sets over a common universe $U$. Then we have the following:

$$
\begin{aligned}
& \text { (i) }(F, A) \vee((G, B) \tilde{\cup}(H, C))= \\
& ((F, A) \vee(G, B)) \tilde{\cup}((F, A) \vee(H, C)) ; \\
& (i i)(F, A) \vee\left((G, B) \cap_{\varepsilon}(H, C)\right)= \\
& ((F, A) \vee(G, B)) \cap_{\varepsilon}((F, A) \vee(H, C)) ; \\
& (\text { iii })(F, A) \vee((G, B) \text { Ó }(H, C))= \\
& ((F, A) \vee(G, B)) \text { Ó }((F, A) \vee(H, C)) \text {; and } \\
& (i v)(F, A) \vee\left((G, B) \cup_{R}(H, C)\right)= \\
& ((F, A) \vee(G, B)) \cup_{R}((F, A) \vee(H, C)) . \\
& \text { Proof (i): Let }(F, A) \vee((G, B) \tilde{\cup}(H, C))
\end{aligned}
$$

$$
\begin{aligned}
& =(F, A) \vee(K, B \cup C) \\
& =(M, A \times(B \cup C))
\end{aligned}
$$

where $\forall \alpha \in B \cup C$, we have

Again, $\forall(\alpha, \beta) \in(A \times(B \cup C))$,

we have

$M(\alpha, \beta)=F(\alpha) \cup K(\beta)$

$$
=\left\{\begin{array}{l}
F(\alpha) \cup G(\beta),(\alpha, \beta) \in A \times(B-C) ; \\
F(\alpha) \cup H(\beta),(\alpha, \beta) \in A \times(C-B) ; \text { and } \\
F(\alpha) \cup\{G(\beta) \cup H(\beta)\},(\alpha, \beta) \in A \times(B \cap C) .
\end{array}\right.
$$

$$
K(\alpha)=\left\{\begin{array}{l}
G(\alpha), \alpha \in B-C ; \\
H(\alpha), \alpha \in C-B ; \text { and } \\
G(\alpha) \cup H(\alpha), \alpha \in B \cap C .
\end{array}\right.
$$

$$
\begin{aligned}
& \text { Let }((F, A) \vee(G, B)) \sim((F, A) \vee(H, C)) \\
& =(I, A \times B) \widetilde{\cup}(J, A \times C) \\
& =(N,(A \times B) \cup(A \times C)) \\
& =(N, A \times(B \cup C)) \\
& \text { where } \forall(\alpha, \beta) \in A \times B, I(\alpha, \beta)=F(\alpha) \cup G(B) \text {; } \\
& \forall(\alpha, \beta) \in A \times C, J(\alpha, \beta)=F(\alpha) \cup H(B) ; \\
& \text { and } \forall(\alpha, \beta) \in A \times(B \cup C) \text {, we have } \\
& N(\alpha, \beta)=\left\{\begin{array}{l}
I(\alpha, \beta),(\alpha, \beta) \in(A \times B)-(A \times C) ; \\
J(\alpha, \beta),(\alpha, \beta) \in(A \times C)-(A \times B) ; \text { and } \\
I(\alpha, \beta) \cup J(\alpha, \beta),(\alpha, \beta) \in(A \times B) \cap(A \times C)= \\
A \times(B \cap C)
\end{array}\right. \\
& =\left\{\begin{array}{l}
F(\alpha) \cup G(\beta),(\alpha, \beta) \in A \times(B-C) ; \\
F(\alpha) \cup H(\beta),(\alpha, \beta) \in A \times(C-B) ; \text { and } \\
{[F(\alpha) \cup G(\beta)] \cup[F(\alpha) \cup H(\beta)]=} \\
F(\alpha) \cup\{G(\beta) \cup H(\beta),(\alpha, \beta) \in A \times(B \cap C)\} .
\end{array}\right.
\end{aligned}
$$

Hence, $M(\alpha, \beta)=N(\alpha, \beta) \forall(\alpha, \beta) \in A \times(B \cup C)$.

Thus (i) holds.

Similarly, (ii) can be proved.

$$
\begin{aligned}
& \text { (iii) } \quad \text { Let }(F, A) \vee((G, B) \mathrm{O}(H, C)) \\
& =(F, A) \vee(L, B \cap C) \\
& =(P, A \times(B \cap C))
\end{aligned}
$$

where $\forall \alpha \in B \cap C$, we have

$L(\alpha)=G(\alpha) \cap H(\alpha)$.

Again, $\forall(\alpha, \beta) \in A \times(B \cap C)$, 


$$
\begin{aligned}
P(\alpha, \beta) & =F(\alpha) \cup L(\beta) \\
& =F(\alpha) \cup\{G(\beta) \cap H(\beta)\} .
\end{aligned}
$$

Let $((F, A) \vee(G, B))$ Ó $((F, A) \vee(H, C))$

$=(I, A \times B) \mathrm{O}(J, A \times C)$

$=(Q,(A \times B) \cap(A \times C))$

$=(Q, A \times(B \cap C))$

where $\forall(\alpha, \beta) \in A \times B, I(\alpha, \beta)=F(\alpha) \cup G(\beta)$;

$\forall(\alpha, \beta) \in A \times C, J(\alpha, \beta)=F(\alpha) \cup H(\beta) ;$

and $\forall(\alpha, \beta) \in A \times(B \cap C)$,

$Q(\alpha, \beta)=I(\alpha, \beta) \cap J(\alpha, \beta)$

$$
\begin{aligned}
& =[F(\alpha) \cup G(\beta)] \cap[F(\alpha) \cup H(\beta)] \\
& =F(\alpha) \cup\{G(\beta) \cap H(\beta)\} .
\end{aligned}
$$

Hence $P(\alpha, \beta)=Q(\alpha, \beta) \forall(\alpha, \beta) \in A \times(B \cap C)$, Thus (iii) holds.

Similarly, (iv) can be proved.

To illustrate Proposition 3.1(i) and (iii), let consider the following example.

\section{Example 3.1}

Let $U=\left\{h_{1}, h_{2}, h_{3}, h_{4}, h_{5}\right\}$ be a universe set consisting of five types of dresses and let $E=\left\{e_{1}, e_{2}, e_{3}, e_{4}\right\}$ be the parameter set, where $e_{i}, i=1,2,3,4$, represents respectively casual, traditional, colourful, and fashionable.

Let $A=\left\{e_{1}, e_{3}\right\}, B=\left\{e_{1}, e_{2}\right\}$ and $C=\left\{e_{2}, e_{4}\right\}$ such that $(F, A)=\left\{\left(e_{1},\left\{h_{1}, h_{2}\right\}\right),\left(e_{3},\left\{h_{3}\right\}\right)\right\} ;$

$(G, B)=\left\{\left(e_{1},\left\{h_{1}\right\}\right),\left(e_{2},\left\{h_{4}\right\}\right)\right\} ;$ and

$(H, C)=\left\{\left(e_{2},\left\{h_{3}, h_{4}\right\}\right),\left(e_{4},\left\{h_{2}\right\}\right)\right\}$.

Let $(F, A) \vee((G, B) \sim(H, C))$

$=(F, A) \vee(K, B \cup C)$

$=(M, A \times(B \cup C))$

where $\forall \alpha \in B \cup C=\left\{e_{1}, e_{2}, e_{4}\right\}$,

$K(\alpha)=\left\{\begin{array}{l}G(\alpha), \text { if } \alpha \in B-C=\left\{e_{1}\right\} ; \\ H(\alpha), \text { if } \alpha \in C-B=\left\{e_{4}\right\} ; \text { and } \\ G(\alpha) \cup H(\alpha), \text { if } \alpha \in B \cap C=\left\{e_{2}\right\} .\end{array}\right.$

and

$\forall(\alpha, \beta) \in A \times(B \cup C)=\left\{\left(e_{1}, e_{1}\right),\left(e_{1}, e_{2}\right),\left(e_{1}, e_{4}\right),\left(e_{3}, e_{1}\right),\left(e_{3}, e_{2}\right),\left(e_{3}, e_{4}\right)\right\}$

$M(\alpha, \beta)=F(\alpha) \cup K(\beta)$

$$
=\left\{\begin{array}{l}
F(\alpha) \cup G(\beta), \alpha \in A=\left\{e_{1}, e_{3}\right\}, \beta \in B-C=\left\{e_{1}\right\} ; \\
F(\alpha) \cup H(\beta), \alpha \in A=\left\{e_{1}, e_{3}\right\}, \beta \in C-B=\left\{e_{4}\right\} ; \text { and } \\
F(\alpha) \cup(G(\beta) \cup H(\beta)), \alpha \in A=\left\{e_{1}, e_{3}\right\}, \beta \in B \cap C=\left\{e_{2}\right\} .
\end{array}\right.
$$

Thus, $\quad M\left(e_{1}, e_{2}\right)=\left\{h_{1}, h_{2}\right\}$;

$$
\begin{aligned}
& M\left(e_{1}, e_{2}\right)=\left\{h_{1}, h_{2}, h_{3}, h_{4}\right\} ; \\
& M\left(e_{1}, e_{4}\right)=\left\{h_{1}, h_{2}\right\} ; \\
& M\left(e_{3}, e_{1}\right)=\left\{h_{1}, h_{3}\right\} ; \\
& M\left(e_{3}, e_{2}\right)=\left\{h_{3}, h_{4}\right\} ; \text { and } \\
& M\left(e_{3}, e_{4}\right)=\left\{h_{2}, h_{3}\right\} . \\
& \text { Let }((F, A) \vee(G, B)) \sim((F, A) \vee(H, C)) \\
& =(I, A \times B) \sim(J, A \times C) \\
& =(N,(A \times B) \cup(A \times C)) \\
& =(N, A \times(B \cup C)) \\
& \text { where } \\
& \forall(\alpha, \beta) \in A \times B=\left\{\left(e_{1}, e_{1}\right),\left(e_{1}, e_{2}\right),\left(e_{3}, e_{1}\right),\left(e_{3}, e_{2}\right)\right\}, \\
& I(\alpha, \beta)=F(\alpha) \cup G(\beta) ; \\
& \forall(\alpha, \beta) \in A \times C=\left\{\left(e_{1}, e_{2}\right),\left(e_{1}, e_{4}\right),\left(e_{3}, e_{2}\right),\left(e_{3}, e_{4}\right)\right\}, \\
& J(\alpha, \beta)=F(\alpha) \cup H(\beta) ; \\
& \quad \forall(\alpha, \beta) \in A \times(B \cup C)= \\
&
\end{aligned}
$$
and

$$
\begin{gathered}
\left\{\left(e_{1}, e_{1}\right),\left(e_{1}, e_{2}\right),\left(e_{1}, e_{4}\right),\left(e_{3}, e_{1}\right),\left(e_{3}, e_{2}\right),\left(e_{3}, e_{4}\right)\right\}, \\
N(\alpha, \beta)=\left\{\begin{array}{l}
F(\alpha) \cup G(\beta), \alpha \in A= \\
\left\{e_{1}, e_{3}\right\}, \beta \in B-C=\left\{e_{1}\right\} ; \\
F(\alpha) \cup H(\beta), \alpha \in A= \\
\left\{e_{1}, e_{3}\right\}, \beta \in C-B=\left\{e_{4}\right\} ; \text { and } \\
(F(\alpha) \cup G(\beta)) \cup(F(\alpha) \cup H(\beta)) \\
=F(\alpha) \cup\{G(\beta) \cup H(\beta)\}, \alpha \in A= \\
\left\{e_{1}, e_{3}\right\}, \beta \in B \cap C=\left\{e_{2}\right\} .
\end{array}\right.
\end{gathered}
$$

It implies

$$
\begin{aligned}
& N\left(e_{1}, e_{1}\right)=\left\{h_{1}, h_{2}\right\} ; \\
& N\left(e_{1}, e_{2}\right)=\left\{h_{1}, h_{2}, h_{3}, h_{4}\right\} ; \\
& N\left(e_{1}, e_{4}\right)=\left\{h_{1}, h_{2}\right\} ; \\
& N\left(e_{3}, e_{1}\right)=\left\{h_{1}, h_{3}\right\} ; \\
& N\left(e_{3}, e_{2}\right)=\left\{h_{3}, h_{4}\right\} ; \text { and }
\end{aligned}
$$$$
\hat{N}\left(e_{3}, e_{4}\right)=\left\{h_{2}, h_{3}\right\} \text {. }
$$

Hence

$M(\alpha, \beta)=N(\alpha, \beta) \forall(\alpha, \beta) \in A \times(B \cup C)$.

Thus $(F, A) \vee((G, B) \tilde{\cup}(H, C))=((F, A) \vee(G, B)) \tilde{\cup}((F, A) \vee(H, C))$.

Let $(F, A) \vee((G, B)$ Ó $(H, C))$

$=(F, A) \vee(L,(B \cap C))=(P, A \times(B \cap C))$

where $\forall \alpha \in B \cap C=\left\{e_{2}\right\}$,

$L(\alpha)=G(\alpha) \cap H(\alpha)$; 
and $\forall(\alpha, \beta) \in A \times(B \cap C)=\left\{\left(e_{1}, e_{2}\right),\left(e_{3}, e_{2}\right)\right\}$, $P(\alpha, \beta)=F(\alpha) \cup L(\beta)=F(\alpha) \cup\{G(\beta) \cap H(\beta)\}$.

Then

$$
\begin{aligned}
P\left(e_{1}, e_{2}\right) & =F\left(e_{1}\right) \cup\left\{G\left(e_{2}\right) \cap H\left(e_{2}\right)\right\} \\
& =\left\{h_{1}, h_{2}\right\} \cup\left\{h_{4}\right\}=\left\{h_{1}, h_{2}, h_{4}\right\} .
\end{aligned}
$$

Similarly, $P\left(e_{3}, e_{2}\right)=\left\{h_{3}, h_{4}\right\}$.

Let $((F, A) \vee(G, B)) O ́((F, A) \vee(H, C))$

$=(I, A \times B)$ Ó $(J, A \times C)$

$=(Q,(A \times B) \cap(A \times C))=(Q, A \times(B \cap C))$

where

$\forall(\alpha, \beta) \in A \times B=\left\{\left(e_{1}, e_{1}\right),\left(e_{1}, e_{2}\right),\left(e_{3}, e_{1}\right),\left(e_{3}, e_{2}\right)\right\}$,

$$
I(\alpha, \beta)=F(\alpha) \cup G(\beta) ;
$$

$\forall(\alpha, \beta) \in A \times C=\left\{\left(e_{1}, e_{2}\right),\left(e_{1}, e_{4}\right),\left(e_{3}, e_{2}\right),\left(e_{3}, e_{4}\right)\right\}$,

$$
J(\alpha, \beta)=F(\alpha) \cup H(\beta) ;
$$

and $\forall(\alpha, \beta) \in A \times(B \cap C)=\left\{\left(e_{1}, e_{2}\right),\left(e_{3}, e_{2}\right)\right\}$, we have

$Q(\alpha, \beta)=I(\alpha, \beta) \cap J(\alpha, \beta)$.

Thus

$Q\left(e_{1}, e_{2}\right)=I\left(e_{1}, e_{2}\right) \cap J\left(e_{1}, e_{2}\right)=\left\{h_{1}, h_{2}, h_{4}\right\} \cap\left\{h_{1}, h_{2}, h_{3}, h_{4}\right\}$

$=\left\{h_{1}, h_{2}, h_{4}\right\} ;$ and

$Q\left(e_{3}, e_{2}\right)=I\left(e_{3}, e_{2}\right) \cap J\left(e_{3}, e_{2}\right)=\left\{h_{3}, h_{4}\right\} \cap\left\{h_{3}, h_{4}\right\}$

$$
=\left\{h_{3}, h_{4}\right\} .
$$

Hence

$P(\alpha, \beta)=Q(\alpha, \beta) \forall(\alpha, \beta) \in A \times(B \cap C)$. This proves (iii).

\section{Proposition 3.2 (Distributive Properties of AND-Operation)}

Let $(F, A),(G, B)$ and $(H, C)$ be soft sets over a common universe $U$. Then the following hold.

(i) $(F, A) \wedge((G, B) \tilde{\cup}(H, C))=((F, A) \wedge(G, B)) \tilde{\cup}((F, A) \vee(H, C))$;

(ii) $(F, A) \wedge\left((G, B) \cap_{\varepsilon}(H, C)\right)=((F, A) \wedge(G, B)) \cap_{\varepsilon}((F, A) \vee(H, C))$;

(iii) $(F, A) \wedge((G, B) O ́(H, C))=((F, A) \wedge(G, B)) O ́((F, A) \wedge(H, C))$; and (iv)

$(F, A) \wedge\left((G, B) \cup_{R}(H, C)\right)=((F, A) \wedge(G, B)) \cup_{R}((F, A) \wedge(H, C))$.

\section{Proof.}

Let $(F, A) \wedge((G, B) \sim(H, C))$

$=(F, A) \wedge(K, B \cup C)$

$=(S, A \times(B \cup C))$

where $\forall \alpha \in(G, B)$, we have
$K(\alpha)=\left\{\begin{array}{l}G(\alpha), \alpha \in B-C ; \\ H(\alpha), \alpha \in C-B ; \text { and } \\ G(\alpha) \cup H(\alpha), \alpha \in B \cap C .\end{array}\right.$

$\forall(\alpha, \beta) \in A \times(B \cup C)$,

$S(\alpha, \beta)=F(\alpha) \cap K(\alpha)$

$=\left\{\begin{array}{l}F(\alpha) \cap G(\beta), \alpha \in A, \beta \in B-C \\ F(\alpha) \cap H(\beta), \alpha \in A, \beta \in C-B, \text { and } \\ F(\alpha) \cap\{G(\beta)\} \cup H(\beta), \alpha \in A, \beta \in B \cap C .\end{array}\right.$

Let $((F, A) \wedge(G, B)) \sim((F, A) \wedge(H, C))$

$=(X, A \times B) \cup(Y, A \times C)$

$=(T,(A \times B) \cup(A \times C))$

$=(T, A \times(B \cup C))$

where $\forall(\alpha, \beta) \in A \times B$, we have

$X(\alpha, \beta)=F(\alpha) \cap G(\beta)$

$\forall(\alpha, \beta) \in A \times C$, we have

$Y(\alpha, \beta)=F(\alpha) \cap H(\beta)$

and $\forall(\alpha, \beta) \in A \times(B \cup C)$, we have

$T(\alpha, \beta)=\left\{\begin{array}{l}X(\alpha, \beta),(\alpha, \beta) \in(A \times B)-(A \times C)=A \times(B-C) ; \\ Y(\alpha, \beta),(\alpha, \beta) \in(A \times C)-(A \times B)=A \times(C-B) ; \text { and } \\ X(\alpha, \beta) \cup Y(\alpha, \beta),(\alpha, \beta) \in(A \times B) \cap(A \times C)=A \times(B \cap C) ;\end{array}\right.$

$$
=\left\{\begin{array}{l}
F(\alpha) \cap G(\beta), \alpha \in A, \beta \in(B-C) ; \\
F(\alpha) \cap H(\beta), \alpha \in A, \beta \in(C-B) ; \text { and } \\
\{F(\alpha) \cap G(\beta)\} \cup\{F(\alpha) \cap H(\beta)\} \\
=F(\alpha) \cap\{G(\beta) \cup H(\beta)\}, \alpha \in A, \beta \in B \cap C .
\end{array}\right.
$$

It follows $S(\alpha, \beta)=T(\alpha, \beta) \forall(\alpha, \beta) \in A \times(B \cup C)$. Hence, (i) is satisfied.

Similarly, (ii) can be proved using the techniques in (i).

Let $(F, A) \wedge((G, B)$ Ó $(H, C))$

$=(F, A) \wedge(L, B \cap C)$

$=(O, A \times(B \cap C))$

where $\forall \alpha \in B \cap C$,

$L(\alpha)=G(\alpha) \cap H(\alpha)$;

and $\forall(\alpha, \beta) \in A \times(B \cap C)$, we have

$O(\alpha, \beta)=F(\alpha) \cap L(\beta)$

$$
=F(\alpha) \cap\{G(\beta) \cap H(\beta)\} .
$$

Let $((F, A) \wedge(G, B))$ Ó $((F, A) \wedge(H, C))$

$=(M, A \times B) \mathrm{O}(N, A \times C)$

$=(R,(A \times B)) \cap(A \times C)=(R, A \times(B \cap C))$

where $\forall(\alpha, \beta) \in A \times B, M(\alpha, \beta)=F(\alpha) \cap G(\beta)$

$\forall(\alpha, \beta) \in A \times C, N(\alpha, \beta)=F(\alpha) \cap H(\beta)$ 
and $\forall(\alpha, \beta) \in A \times(B \cap C)$,

$R(\alpha, \beta)=M(\alpha, \beta) \cap N(\alpha, \beta)$

$$
\begin{aligned}
& =\{F(\alpha) \cap G(\beta)\} \cap\{F(\alpha) \cap H(\beta)\} \\
& =F(\alpha) \cap\{G(\beta) \cap H(\beta)\} .
\end{aligned}
$$

Hence $O(\alpha, \beta)=R(\alpha, \beta) \forall(\alpha, \beta) \in A \times(B \cap C)$

which completes the proof of (iii).

Similarly, (iv) can be proved.

Let us now illustrate (iv) in example 3.2 below by considering the soft sets in example 3.1.

\section{Example 3.2}

$$
\begin{aligned}
& \text { Let }(F, A) \wedge\left((G, B) \cup_{R}(H, C)\right) \\
& =(F, A) \wedge(K, B \cap C)=(T, A \times(B \cap C)) \\
& \text { where } \forall \alpha \in B \cap C=\left\{e_{2}\right\}, \\
& K(\alpha)=G(\alpha) \cup H(\alpha) ; \\
& \text { and } \forall(\alpha, \beta) \in A \times(B \cap C)=\left\{\left(e_{1}, e_{2}\right),\left(e_{3}, e_{2}\right)\right\}, \\
& T(\alpha, \beta)=F(\alpha) \cap K(\beta)=F(\alpha) \cap\{G(\beta) \cup H(\beta)\} . \\
& \text { Thus, } \\
& T\left(e_{1}, e_{2}\right)=F\left(e_{1}\right) \cap\left\{G\left(e_{2}\right) \cup H\left(e_{2}\right)\right\} ; \\
& \qquad=\left\{h_{1}, h_{2}\right\} \cap\left\{h_{3}, h_{4}\right\}=\varnothing ; \text { and } \\
& T\left(e_{3}, e_{2}\right)=\left\{h_{3}\right\} \cap\left\{h_{3}, h\right\}=\left\{h_{3}\right\} . \\
& \text { Let }((F, A) \wedge(G, B)) \cup_{R}((F, A) \wedge(H, C)) \\
& =(I, A \times B) \cup_{R}(J, A \times C) \\
& =(P,(A \times B) \cap(A \times C))=(P, A \times(B \cap C))
\end{aligned}
$$

$$
\begin{aligned}
& \text { where } \\
& \forall(\alpha, \beta) \in A \times B=\left\{\left(e_{1}, e_{1}\right),\left(e_{1}, e_{2}\right),\left(e_{3}, e_{1}\right),\left(e_{3}, e_{2}\right)\right\}, \\
& I(\alpha, \beta)=F(\alpha) \cap G(\beta) ; \\
& \forall(\alpha, \beta) \in A \times C=\left\{\left(e_{1}, e_{2}\right),\left(e_{1}, e_{4}\right),\left(e_{3}, e_{2}\right),\left(e_{3}, e_{4}\right)\right\}, \\
& J(\alpha, \beta)=F(\alpha) \cap H(\beta) ; \\
& \text { and } \forall(\alpha, \beta) \in A \times(B \cap C)=\left\{\left(e_{1}, e_{2}\right),\left(e_{3}, e_{2}\right)\right\}, \\
& P(\alpha, \beta)=I(\alpha, \beta) \cup J(\alpha, \beta) . \\
& \text { Thus } \\
& P\left(e_{1}, e_{2}\right)=I\left(e_{1}, e_{2}\right) \cup J\left(e_{1}, e_{2}\right)=\varnothing ; \\
& \text { and } P\left(e_{3}, e_{2}\right)=I\left(e_{3}, e_{2}\right) \cup J\left(e_{3}, e_{2}\right)=\left\{h_{3}\right\} . \\
& \text { Hence } T(\alpha, \beta)=P(\alpha, \beta) \forall(\alpha, \beta) \in A \times(B \cap C) \\
& \text { which satisfies (iv). }
\end{aligned}
$$

\section{Proposition 3.3}

Let $(F, A),(G, B)$ and $(H, C)$ be soft sets over a common universe $U$. Then the following hold.

$$
\begin{aligned}
& (i)(F, A) \wedge\left((G, B) \smile_{R}(H, C)\right)= \\
& ((F, A) \wedge(G, B)) \smile_{R}((F, A) \wedge(H, C)) \\
& (i i)\left((F, A) \smile_{R}(G, B)\right) \wedge(H, C)= \\
& \left((F, A) \wedge(H, C) \smile_{R}((G, B) \wedge(H, C))\right. \\
& (i i i)(F, A) \vee\left((G, B) \smile_{R}(H, C)\right) \neq \\
& ((F, A) \vee(G, B)) \smile_{R}((F, A) \vee(H, C)) \\
& (i v)\left((F, A) \smile_{R}(G, B)\right) \vee(H, C) \neq \\
& ((F, A) \vee(H, C)) \smile_{R}((G, B) \vee(H, C))
\end{aligned}
$$

\section{Proof.}

(i)Let $(F, A) \wedge\left((G, B)_{R}(H, C)\right)$

$=(F, A) \wedge(K, B \cap C)$

$=(L, A \times(B \cap C))$

where $\forall \alpha \in B \cap C, K(\alpha)=G(\alpha)-H(\alpha)$;

and $\forall(\alpha, \beta) \in A \times(B \cap C), L(\alpha, \beta)=F(\alpha) \cap K(\beta)$

$=F(\alpha) \cap\{G(\beta)-H(\beta)\}$.

Again, let $((F, A) \wedge(G, B))_{R}((F, A) \wedge(H, C))$

$=(M, A \times B) \smile_{R}(N, A \times C)$

$=(P,(A \times B) \cap(A \times C))$

$=(P, A \times(B \cap C))$

where $\forall(\alpha, \beta) \in A \times B, M(\alpha, \beta)=F(\alpha) \cap G(\beta)$; $\forall(\alpha, \beta) \in A \times C, N(\alpha, \beta)=F(\alpha) \cap H(\beta)$; and $\forall(\alpha, \beta) \in A \times(B \cap C)$,

$$
\begin{aligned}
P(\alpha, \beta) & =M(\alpha, \beta)-N(\alpha, \beta) \\
& =[F(\alpha) \cap G(\beta)]-[F(\alpha) \cap H(\beta)] \\
& =F(\alpha) \cap\{G(\beta)-H(\beta)\} .
\end{aligned}
$$

Hence $L(\alpha, \beta)=P(\alpha, \beta)$.Thus (i) holds.

Similar argument in (i) can be used to prove (ii). The proof is therefore omitted.

$$
\begin{aligned}
& \text { (iii) } \quad \text { Let }(F, A) \vee\left((G, B) \smile_{R}(H, C)\right) \\
& =(F, A) \vee(K, B \cap C) \\
& =(K, A \times(B \cap C)) \\
& \text { where } \forall \alpha \in B \cap C, \quad L(\alpha)=G(\alpha)-H(\alpha) ; \\
& \text { and } \forall(\alpha, \beta) \in A \times(B \cap C), \\
& \qquad \begin{aligned}
& K(\alpha, \beta)=F(\alpha) \cup L(\beta) \\
&= F(\alpha) \cup[G(\beta)-H(\beta)] \\
& \smile_{R}
\end{aligned}
\end{aligned}
$$




$$
\begin{aligned}
& \text { Let }((F, A) \vee(G, B)) \quad((F, A) \vee(H, C)) \\
& =(I, A \times B) \smile_{R}(J, A \times C) \\
& =(R,(A \times B) \cap(A \times C)) \\
& =(R, A \times(B \cap C)) \\
& \text { where } \forall(\alpha, \beta) \in A \times B \text {, } \\
& I(\alpha, \beta)=F(\alpha) \cup G(\beta) \text {; } \\
& \forall(\alpha, \beta) \in A \times C \text {, } \\
& J(\alpha, \beta)=F(\alpha) \cup H(\beta), \\
& \text { and if }(\alpha, \beta) \in A \times(B \cup C) \text {, } \\
& R(\alpha, \beta)=I(\alpha, \beta)-J(\alpha, \beta) \\
& =[F(\alpha) \cup G(\beta)]-[F(\alpha) \cup H(\beta)] \\
& \neq F(\alpha) \cup[G(\beta)-H(\beta)] \text {. }
\end{aligned}
$$

Hence $\quad(K, A \times(B \cap C)) \neq(R, A \times(B \cap C))$, which proves (iii).

Using similar arguments as in (iii), (iv) can be proved.

\section{Remark}

The operations of union, intersection or difference (both restricted and extended) do not distribute over OR-operation or AND-operation. For example, $(F, A) \sim((G, B) \wedge(H, C))=(L, A \cup(B \times C))$ does not hold as $A \cup(B \times C)$ does not exist.

\section{CONCLUSION}

The distributive properties of AND $(\wedge)$ and OR $(\vee)$ operations with respect to $\tilde{\cup}, \cup_{R}, \cap_{\varepsilon} \smile_{R}$ Ó and operations are presented. It is observed that the operations $\tilde{\cup}, \cup_{R}, \cap \varepsilon, \quad \dot{O}$ and do not distribute over the operations $\wedge$ and $\vee$.

\section{REFERENCES}

[1] Ali, M.I., Feng, F.,Liu, X., Min, W.R,,Shabir M. 2009. On Some New Operations on Soft Set Theory, Computers and Mathematics with Applications, 57 , 1547-1553.

[2] Fu, Li. 2011. Notes on the Soft Operations, ARPN Journal of Systems and Software,1(6),205-208.

[3] Ge,X., and Yang, S. 2011. Investigations on some Operations of Soft Sets, World Academy of sciences, Engineering andTechnology,75,1113-1116

[4] Maji, P.K., Biswas, R., Roy,A.R. 2003. Soft Set Theory, Computers and Mathematics with Applications, 45, 555562.

[5] Molodtsov,D. 1999. Soft Set Theory- First Results, Computers and Mathematics with Applications, 3,19-31.

[6] Neog, I.J and Sut, D.K.. 2011. A new Approach to the Theory of Soft Set,International Journal of Computer Applications ,(0975-8887),32(2).

[7] Qin, K and Hong Z. 2010.On Soft Equality ,Journal of Computational and Applied Mathematics,234,13471355.

[8] Sezgin, A. and Atagun A.O. 2011.On Operation of Soft Set,Computers and Mathematics with Applications,61, 1457-1467

[9] Zhu, P. and Wen, Q.2012. Operations on Soft Sets revisited, submitted for publication(CoRRabs/1205.2887). 